\title{
TDChain: uso de blockchain na cadeia de distribuição de medicamentos
}

\author{
Jhemeson Silva Mota ${ }^{1}$, Ian Macedo Maiwald Santos ${ }^{1}$, Alexandre Henrique Kavalerski \\ Teixeira ${ }^{1}$, Kennedy Santos Torres ${ }^{1}$, Madianita Bogo Marioti ${ }^{1}$ \\ ${ }^{1}$ Departamento de Computação - Centro Universitário Luterano de Palmas (CEULP/ULBRA) Palmas, \\ TO - Brasil \\ \{jhemesonmotta, ianmaiscedo, kavalerskialexandre, altmaisefequeatro, \\ madianitab\}@gmail.com
}

\begin{abstract}
Resumo. O Brasil ocupa a sexta posição no ranking dos maiores consumidores de medicamentos do mundo. Com o aumento da procura por medicamentos surge a oportunidade para que produtos clandestinos e sem qualidade se misturem entre os legítimos em pontos de distribuição como, por exemplo, farmácias. Blockchain pode ser entendido como uma solução de banco de dados distribuídos que mantém uma lista crescente de registros confirmados pelos nós participantes. O presente trabalho apresenta a TDChain, uma proposta de uso de Blockchain que registra o transporte de um medicamento desde o fabricante até o seu destino final para facilitar a identificação de medicamentos falsificados.
\end{abstract}

\section{Introdução}

O Conselho Federal de Farmácia (2016) aponta que o Brasil se encontra como uma potência do mercado farmacêutico mundial, uma vez que ocupa a sexta posição entre os maiores mercados consumidores de medicamentos no mundo, atrás de países como China, Japão e Estados Unidos.

O envelhecimento da população brasileira é considerado um dos principais fatores para o aumento no consumo de remédios, algo que se intensifica com o passar dos anos (CONSELHO FEDERAL DE FARMÁCIA, 2016; MAGALHÃES et al., 2018). Com o aumento na venda de medicamentos, tem-se uma oportunidade para que produtos clandestinos e sem garantia de qualidade, segurança e eficácia se misturem entre os legítimos.

De acordo com a Organização Mundial de Saúde (2013), um medicamento que não possui uma embalagem e etiqueta adequadas, que não respeita a sua identidade ou fonte, ou com alterações em sua fórmula original são classificados como falsificados. Segundo a Anvisa (2019), os medicamentos falsificados podem ser produzidos no Brasil ou podem vir de países que fazem fronteira com o Brasil.

Yli-Huumo et al. (2016) definem Blockchain como uma solução de banco de dados distribuídos que mantém uma lista crescente de registros de dados que são confirmados pelos nós participantes. Os dados, nesta solução, são armazenados em um registro público que inclui informações de todas as transferências de dados já concluídas. De acordo com Michael, Cohn e Butcher (2018), o Blockchain oferece um mecanismo de validação por consenso através de uma rede de computadores que facilita as transações peer-to-peer sem a necessidade de uma autoridade intermediária ou centralizada para atualizar e manter as informações geradas pelas transações.

O conceito Blockchain foi desenvolvido por Satoshi Nakamoto com a finalidade de ser aplicado em sistema monetário, especificamente para ser utilizado na criptomoeda Bitcoin (NAKAMOTO, 2008). No entanto, o uso de Blockchain pode ser utilizado - e tem sido aliado a diferentes tecnologias para diversas finalidades. Joselli (2018) apresenta em seu trabalho pontos positivos para a integração de jogos com Blockchain e descreve o funcionamento do jogo CriptoKitties, uma das primeiras tentativas de implantar a tecnologia para fins de lazer. 
O presente trabalho visa uma explanação dos conceitos acerca do Blockchain e como estes podem ser aplicados ao ramo farmacêutico no processo de identificação de medicamentos falsificados, bem como os desafios para a implantação de tal aplicação. A ferramenta proposta, intitulada TDChain, tem como objetivo permitir o rastreamento do caminho do remédio que é encontrado na farmácia - desde o fabricante até o seu destino final.

\section{Fundamentação teórica}

\subsection{Blockchain}

Christidis e Devetsikiotis (2016) afirmam que uma blockchain é uma estrutura de dados que é replicada e compartilhada entre os membros de uma rede. Nakamoto (2008) a introduz com o Bitcoin como meio para solucionar o problema do gasto-duplo. Como resultado de como os nós na rede Bitcoin (os chamados mineradores) anexam transações validadas, mutuamente acordadas, a blockchain do Bitcoin abriga a razão geral das transações que estabelece quem possui o que (ERIS INDUSTRIES, 2016).

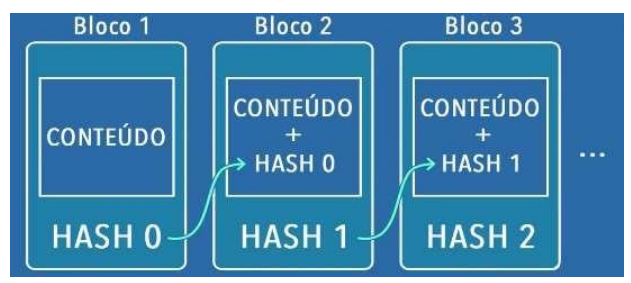

Figura 1. Relação entre blocos na blockchain.

Fonte: Prado (2017).

Em uma Blockchain, cada transação é validada e, juntamente com um grupo de outras transações também validadas, é adicionada como um novo Bloco a uma cadeia de transações já existente (como pode ser observado na Figura 1), dando origem ao nome que em tradução literal significa "cadeia de blocos" (MICHAEL; COHN; BUTCHER, 2018). Uma vez que uma transação tenha sido adicionada à cadeia, esta não pode ser alterada ou removida.

Isso estabelece um link entre os blocos, criando assim uma cadeia de blocos ou blockchain. Qualquer nó com acesso a essa lista de blocos ordenados e vinculados pode lê-la e descobrir qual é o estado universal dos dados que estão circulando na rede (ERIS INDUSTRIES, 2016; ANTONOPOULOS, 2014).

Segundo Christidis e Devetsikiotis (2016), entende-se melhor como funciona uma blockchain, ao examinar como uma rede blockchain é executada. Os autores descrevem que esta rede é um conjunto de nós (clientes) que operam no mesmo blockchain através da cópia que cada um possui e, com isso, um nó geralmente pode atuar como um ponto de entrada para vários usuários blockchain diferentes na rede, mas, por simplicidade, assume-se que cada usuário realiza uma transação na rede através de seu próprio nó. Esses nós formam uma rede peer-to-peer, onde:

1. Os usuários interagem com o blockchain por meio de um par de chaves (MICROSOFT, 2014).

2. Os pares vizinhos garantem que esta transação seja válida antes de retransmiti-la; transações inválidas são descartadas. Eventualmente, essa transação é espalhada por toda a rede.

3. As transações que foram coletadas e validadas pela rede usando o processo acima durante um intervalo de tempo acordado, são ordenadas e encapsuladas em um bloco candidato com registro de data e hora. Este é um processo chamado de mineração.

4. Os nós verificam se o bloco sugerido contém transações válidas, e referência via hash o bloco anterior correto em sua cadeia. Se esse for o caso, adiciona-se o bloco à 
cadeia e apresentam as transações que ele contém para atualizar sua visão de mundo. Se esse não for o caso, o bloco proposto é descartado. Isso marca o fim de uma rodada.

\subsection{Smart contracts}

De acordo com Venegas (2017), smart contracts são contratos computáveis automatizados que são executados em blockchains. $O$ autor acrescenta que em um cenário ideal há a necessidade de automatizar os fluxos de trabalhos e/ou eliminar centros de custo (intermediários) enquanto tem-se acesso concorrente a uma versão organizacionalmente descentralizada (fisicamente separado), mas logicamente centralizada (única) da verdade.

Rande (2018) diz que um Smart Contract é um código de computador auto executável, sem qualquer interface com o usuário, que pode definir regras e consequências para o não cumprimento destas, tal como um contrato tradicional.

Tais contratos, de acordo com Gonçalves e Camargos (2017), permitem que indivíduos acordem entre si transações de bens e valores associados à blockchain que seriam automaticamente executadas assim que as condições contratuais escolhidas fossem cumpridas.

A tecnologia tem como empecilho parcial para sua adoção os requerimentos tradicionais do direito contratual vigente na maioria das jurisdições, sendo que, uma vez assinados, os contratos inteligentes se cumprem de forma automática, independentes dos poderes jurisdicionais tradicionais do Estado. Sendo assim, em alguns contextos específicos, mesmo uma ordem judicial de alto nível teria pouca eficácia para reverter o negócio jurídico realizado através de um smart contract (GONÇALVES; CAMARGOS, 2017).

\subsection{Cadeia de distribuição de medicamentos no Brasil}

De acordo com Pinto (2016), a distribuição de medicamentos corresponde a etapas da cadeia logística que engloba características como instalações de armazenagem, layout dos locais de distribuição e métodos de localização dos itens e tipos de distribuição, visando assegurar a qualidade do medicamento e disponibilidade para todos os locais de atendimento.

Ainda para Pinto (2016), a distribuição é a etapa que gera os maiores problemas, especialmente quando não há uma relação de confiança entre o usuário e o administrador da distribuição, uma vez que sistemas de distribuição não regulados podem levar ao surgimento de estoques periféricos. Por este motivo a movimentação deve ser monitorada por um sistema confiável, atualizado e parametrizado.

A Anvisa (2017) aponta, dentre outros, três atores principais que participam da cadeia de distribuição de medicamentos no Brasil:

- Detentor de registro: fabricante ou importador, registra medicamentos de uso humano;

- Distribuidor: armazena e movimenta o medicamento como intermediário entre fabricante e dispensador;

- Dispensador: Farmácias, drogarias, hospitais. Estabelecimentos que fornecem medicamentos, remunerados ou gratuitos, ao consumidor ou paciente. Em 2016 existiam mais de 82 mil farmácias e drogarias no Brasil (CONSELHO FEDERAL DE FARMÁCIA, 2019).

Há também o consumidor, a pessoa que adquire um medicamento em local regularizado à dispensação. Entretanto, a Anvisa o considera parte da etapa de pós- consumo, não sendo membro da cadeia de movimentação de medicamento. 


\section{Materiais e métodos}

Para a definição do problema que o presente trabalho propõe resolver, bem como dos elementos que compõem a proposta de solução, atividades-chave foram realizadas. Tais atividades podem ser vistas na Figura 2 e serão melhor descritas a seguir.

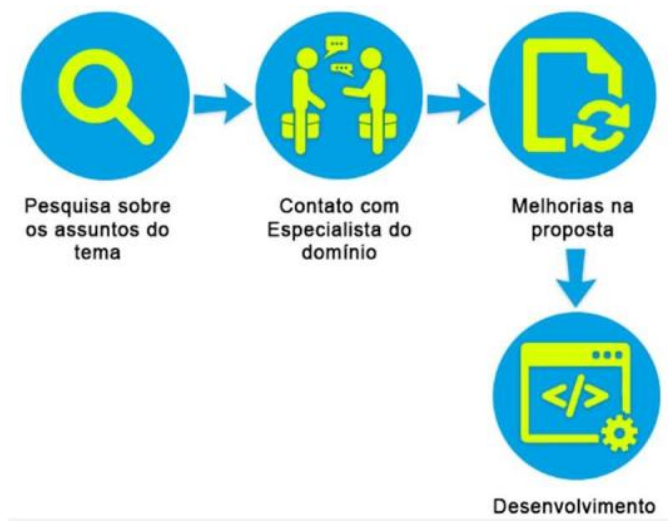

Figura 2. Metodologia do projeto

Visando reunir evidências e estudos relacionados às tecnologias elencadas como fonte de observação, bem como identificar possíveis sugestões de investigação e/ou lacunas na presente pesquisa, foi proposta uma revisão de literatura com mapeamento sistemático como uma etapa fundamental deste trabalho. A revisão foi produzida por dois dos autores do trabalho sob supervisão da orientadora. A partir desta revisão, foi possível vislumbrar um gap de propostas voltadas a utilizar Blockchain no contexto farmacêutico, e por isto, o presente trabalho visa tal área.

Posteriormente, foram realizadas reuniões com um especialista de domínio para validar insights obtidos a partir da revisão de literatura. $\mathrm{O}$ especialista de domínio é dono de uma rede de farmácias na cidade de Palmas-TO e se dispôs a colaborar com o trabalho, tanto validando algumas informações (do ponto de vista de quem trabalha na área) quanto sugerindo melhorias e informando os dados necessários a serem considerados na proposta.

Após estas reuniões, a proposta passou por melhorias com base em feedbacks recebidos do especialista. Importante ressaltar que, até esta etapa do projeto, o que se refere à proposta aborda apenas a parte conceitual desta, ou seja, ainda não existia, até então, nada implementado (que só ocorreu na última etapa demonstrada na Figura 2).

Como última etapa do projeto, ocorreu a implementação da ferramenta proposta. A ferramenta intitulada TDChain é uma dApp (Decentralized Application - ou "Aplicação Descentralizada" em tradução literal), utiliza Blockchain para armazenamento dos dados e tem seu funcionamento descrito de forma mais detalhada na próxima seção.

Para o desenvolvimento da parte visual da aplicação, foi utilizado HTML5, CSS3 e a view engine EmbeddedJS, que possibilita a utilização de variáveis Javascript na construção de uma página de hipertexto. O código existente por trás da página foi todo escrito em Javascript através do Node.Js. Inclusive o Blockchain e os Smart Contracts foram implementados com esta tecnologia. Mais detalhes sobre isto podem ser encontrados na seção seguinte.

Para implementar a comunicação Peer to Peer (P2P) foi utilizada a biblioteca gratuita PeerJS, que gera um ID para cada instância de ponto conectada e provê mecanismos para que um ponto acesse outro.

\section{Resultados e discussões}

Tal como pode ser visto na Figura 3, a arquitetura deste sistema pode ser observada em três camadas. A primeira camada diz respeito ao usuário, este se comunicará com o sistema (TDChain) que realizará todas as operações relacionadas à lógica de negócio e, para 
gravação de dados será utilizado - ao invés de um banco de dados tradicional, como em sistemas tradicionais - o blockchain.

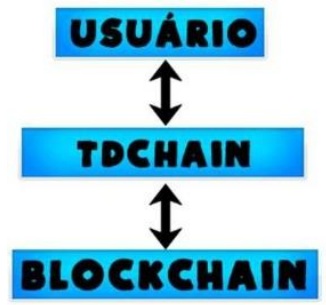

Figura 3. Arquitetura da aplicação.

Existem neste sistema, três tipos de usuários, sendo eles: Fabricante, Farmácia e Distribuidor. No que se refere à permissões em funcionalidades, apenas o usuário Fabricante terá permissões diferentes. Este será o único que pode criar novosmedicamentos no sistema. Os demais poderão apenas enviar os medicamentos que forem de sua propriedade, de tal forma, utilizando Smart Contract de "lei de propriedade": busca garantir que apenas o proprietário de um determinado token digital (no caso, o medicamento) possa operar sobre ele.

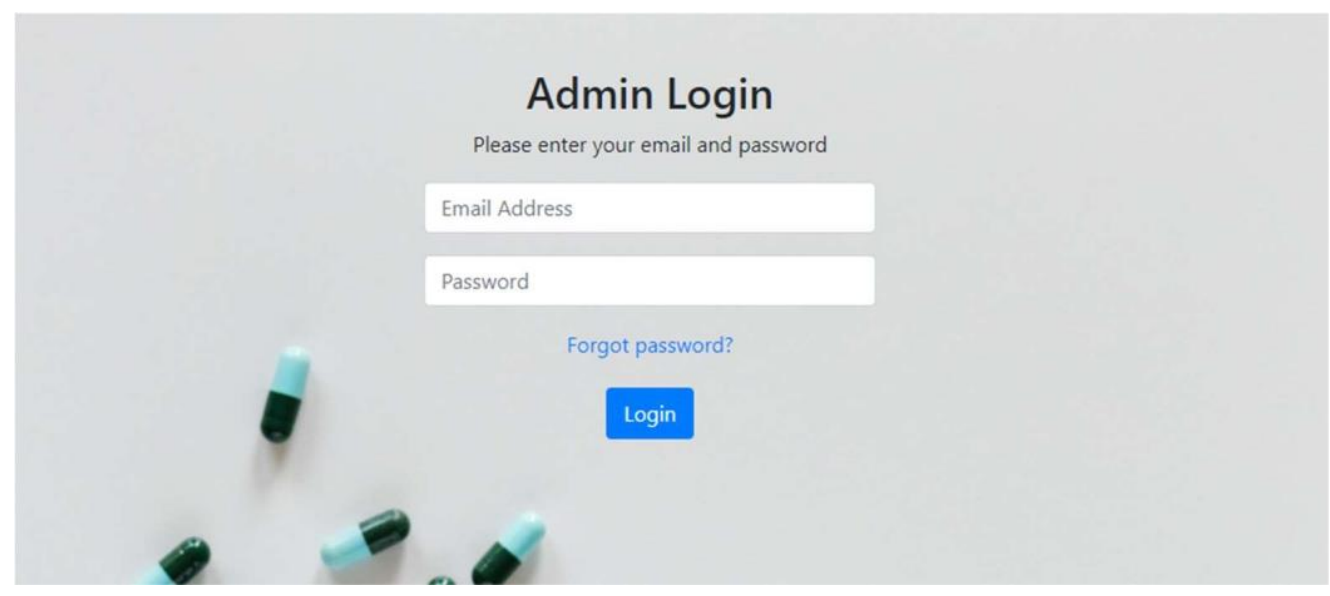

Figura 4. Tela de login do TDChain.

A Figura 4 mostra a tela de login, onde os usuários entrarão com suas credenciais e serão identificados de acordo com seu tipo.

\subsection{Criar Remédio}

O usuário cadastrado no sistema como Fabricante, após efetuar com sucesso o login, visualiza a página inicial do sistema onde poderá utilizar as funcionalidades a seguir: Criar Remédio, Consulta de Precedência, Listar Remédios e Enviar Remédios.

A funcionalidade de Criar Remédio (Figura 5) disponibiliza um formulário onde o Fabricante fornecerá algumas informações a respeito do medicamento a ser criado. O usuário deverá informar o nome do produto que ele pretende criar, podendo ter quantos caracteres for necessário além de ser possível utilizar letras e números. Outra informação que o usuário deverá informar ao preencher o formulário de criação de remédio é a origem deste, podendo ser natural (medicamentos de origem natural), vegetal (medicamentos extraídos de plantas), animal (extraídos de animais), mineral (extraídos de minerais) ou sintético (produzidos por reação química e em laboratórios).

Os dois últimos campos do formulário são sobre a composição do remédio e sobre o local de ação. As opções de local de ação do remédio podem ser: a ação local, que apresentam ação diretamente sobre o local de aplicação. O outro local de ação, o sistêmico é o que funciona somente quando entra em contato com o sangue. Abaixo é exibido o formulário que o Fabricante deve preencher para poder criar um remédio no sistema. 
A última opção que o fabricante de remédio deve selecionar no formulário (Figura 5) é a via de administração do produto a ser criado. As drogas podem ser administradas por diversas vias do corpo, a escolha da via a ser utilizada depende tanto do tipo de droga quanto do paciente. As vias (opções) do formulário são as Parentais e Enterais, sendo as parentais, vias utilizadas para injeção de remédios, que vão diretamente para o tecido ou sangue do paciente. E as vias enterais, as utilizadas por remédios que devem ser ingeridos via trato digestivo.

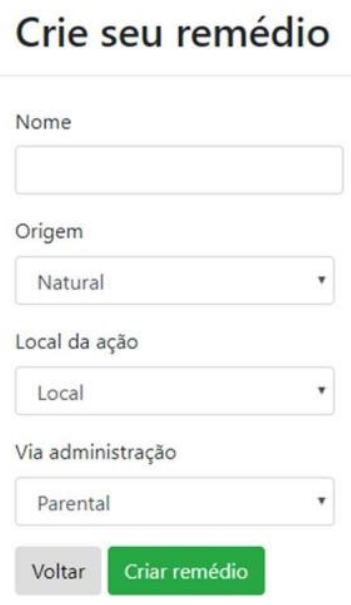

Figura 5. Formulário para criação de remédios.

Ao criar um remédio, será adicionado ao blockchain um bloco contendo um index (número inteiro correspondente à posição do bloco dentro do blockchain); o hash do bloco anterior (algoritmo que mapeia dados de comprimento variável para dados de comprimento fixo), todavia, se este for o primeiro bloco da cadeia, este atributo ficará vazio; o timestamp que guardará data e hora que o bloco foi criado e um atributo chamado data, que armazenará, neste contexto, uma instância da classe Transacao. Esta classe, por sua vez, guardará os seguintes atributos:

- entidadeRemetente: guardará o usuário que enviou o medicamento - no caso de criação, será um valor nulo;

- entidadeDestinatario: representa o usuário que terá a posse do medicamento após a transação;

- medicamento: representa o medicamento em si;

- data: representa data e hora da criação desta instância de Transação.

\subsection{Listar Remédios}

Esta funcionalidade lista os remédios que que existem cadastrados no sistema e serve para fins de controle, conferência e testes.

\section{Medicamentos Existentes}

\begin{tabular}{lllll}
\hline ID & Nome & Local & Via & Dono \\
\hline $6100 \mathrm{c} 21 \mathrm{e} 0882 \mathrm{f} 1 \mathrm{c} 77260 \mathrm{fe} 20 \mathrm{a} 7 \mathrm{a} 6 \mathrm{fa} 9430 \mathrm{a} 9 \mathrm{f3fd}$ & dipirona & Local & Parental & Alexandre
\end{tabular}

Figura 6. Tela de Listagem 
A Figura 6 demonstra a listagem sendo que nesta serão exibidas, para cada medicamento existente, o ID, o nome, o local de Ação, a Via de Administração e o atual dono deste.

\subsection{Enviar Remédio}

O envio de remédios só pode ocorrer caso o usuário remetente tenha o remédio em questão como sua propriedade. O controle dos estoques é feito com a blockchain. A Figura 7 demonstra o formulário que o usuário deverá preencher para poder efetuar o envio de algum remédio para outra entidade.

\section{Envie seu remédio}

\begin{tabular}{l} 
Remédio \\
dipirona \\
Destinatário \\
Jhemeson \\
Voltar Enviar remédio \\
\hline
\end{tabular}

Figura 7. Tela de Envio de Remédio.

Assim como ocorre ao criar um novo remédio, ao enviar um Remédio, será criado um novo bloco na cadeia de blocos com as mesmas informações previamente descritas.

\subsection{Consulta de Precedência}

Outra funcionalidade do sistema é a de Consulta de Precedência. Essa funcionalidade possibilita que o usuário pesquise utilizando o ID de um remédio, todo o caminho que o remédio pesquisado percorreu. Destaca-se que esta é a única funcionalidade do sistema que não requer um usuário logado para funcionar, de tal forma, esta poderá ser acessada pelos usuários que comprarem um remédio em uma farmácia.

Consulte a precedência de algum remédio

$$
\text { Insira o ID do remédio }
$$$$
\text { Pesquisar Itens }
$$

Figura 8. Tela de Pesquisa de Precedência de remédio.

Ao digitar um id válido e clicar em "Pesquisar", o usuário será redirecionado para uma página que contém a tabela que representa o caminho trilhado pelo remédio até o presente momento. Este caminho é exposto na Figura 9.

\section{Consulte a precedência de algum remédio}

\begin{tabular}{|c|c|c|c|c|c|}
\hline Remetente & Endereço & Destinatário & Endereço & Medicamento & Data \\
\hline Criou Remédio & Criou Remédio & $\begin{array}{l}\text { Jhemeson - } \\
\text { Fabricante }\end{array}$ & $\begin{array}{l}1503 \text { S, Palmas- } \\
\text { TO }\end{array}$ & dipirona & $\begin{array}{l}\text { Tue Dec } 112018 \text { 09:06:50 GMT-0300 (Hora oficial } \\
\text { do Brasil) }\end{array}$ \\
\hline $\begin{array}{l}\text { Jhemeson - } \\
\text { Fabricante }\end{array}$ & $\begin{array}{l}1503 \text { S, Palmas- } \\
\text { TO }\end{array}$ & $\begin{array}{l}\text { Alexandre - } \\
\text { Farmácia }\end{array}$ & $\begin{array}{l}506 \text { S, Palmas- } \\
\text { TO }\end{array}$ & dipirona & $\begin{array}{l}\text { Tue Dec } 112018 \text { 09:09:13 GMT-0300 (Hora oficial } \\
\text { do Brasil) }\end{array}$ \\
\hline
\end{tabular}


Desta forma, o usuário poderá conferir de maneira segura se a farmácia que ele comprou o medicamento é, de fato, a que deve possuir o medicamento e, da mesma forma, poderá conferir todos os lugares e datas que o medicamento já esteve.

\section{Conclusões}

O presente trabalho teve como objetivos demonstrar a possibilidade de uso do blockchain no sistema farmacêutico e desenvolver o protótipo de uma solução. A solução proposta visa facilitar a identificação de remédios falsificados possibilitando consultar a precedência de tal produto. Com isto, pode-se considerar que objetivo do trabalho foi alcançado.

O protótipo desenvolvido pode ser identificado como um híbrido no que tange à descentralização. Tal conclusão é possível pois, apesar de ter a base de dados referente às transações (mudanças de precedência de determinado remédio) compartilhada entre todos os nós da rede, o controle de usuários - fabricantes e farmácia, por exemplo - é feito de forma centralizada, pois cada tipo de usuário tem funções específicas dentro da aplicação desenvolvida. Como exemplo do que foi citado, somente usuários fabricantes cadastram novos remédios.

Além disto, a TDChain carece de uma definição mais coesa no que tange ao processo de mineração. Atualmente a figura do minerador não está definida, com isso, todo nó da rede executa automaticamente os algoritmos de mineração, processo computacionalmente pesado que pode acarretar em problemas futuros que afetem a escalabilidade da solução.

Como trabalhos futuros, sugere-se o incremento do sistema TDChain apresentado neste trabalho com uma proposta de mecanismo de mineração, bem como a definição de recompensa quando o minerador encontra o hash ideal de um bloco.

Quanto ao potencial da TDChain e suas vantagens aplicadas ao sistema farmacêutico, deve-se avaliar posteriormente se o sistema desenvolvido pode de fato resolver o problema para o qual se propõe. Para isso, sugere-se o uso da TDChain em ambiente real (visto que, o escopo deste trabalho define sua execução somente em ambiente simulado), bem como monitoramento e análise dos resultados de tal aplicação.

\section{Referências}

ANTONOPOULOS, Andreas M. Mastering Bitcoin: Unlocking Digital Cryptocurrencies. [s.1.]: O'reilly Media, 2014. 298 p.

ANVISA. Especificação de Requisitos, Padrões e Interfaces para o Sistema Nacional de Controle de Medicamentos (SNCM). [s.1.]: Anvisa, 2017. 224 p. Disponível em: <https://goo.gl/r2ouVj>. Acesso em: 10 dez. 2018.

ANVISA. Medicamentos falsificados. Disponível em: <https://goo.gl/oLXYDF>. Acesso em: 08 dez. 2018.

CONSELHO FEDERAL DE FARMÁCIA. Farmácias de elite. 2016. Disponível em: <http://www.cff.org.br/noticia.php?id=3879>. Acesso em: $06 \mathrm{dez}$. 2018. CONSELHO FEDERAL DE FARMÁCIA. DADOS 2016. Disponível em: <https://goo.gl/awZ2c3>. Acesso em: 10 dez. 2018.

CHRISTIDIS, Konstantinos; DEVETSIKIOTIS, Michael. Blockchains and Smart Contracts for the Internet of Things. Ieee Access, [s.1.], v. 4, n. 3, p. 2292-2303, maio 2016. Institute of Electrical and Electronics Engineers (IEEE). Disponível em: <https://ieeexplore.ieee.org/document/7467408>. Acesso em: 24 nov. 2018.

ERIS INDUSTRIES. Eris Industries Documentation: Blockchains. [s.1.]: Eris Industries, 2016. Disponível em: <https://docs.erisindustries.com/explainers/blockchains>. Acesso em: 18 nov. 2018. 
GONÇALVES, P. V. R.; CAMARGOS, R. C. Blockchain, Smart Contracts e "Judge as a Service" no Direito Brasileiro. In: II Seminário Governança das Redes e o Marco Civil da Internet: globalização, tecnologias e conectividade. Anais... Belo Horizonte: Instituto de Referência em Internet e Sociedade - IRIS, 2017. p. 207-212.

JOSELLI, Mark. Blockchain e Games. In: SBGAMES, 17., 2018, Foz do Iguaçu. Proceedings... . Foz do Iguaçu: Sbc, 2018. p. 1 - 11. Disponível em: <https://goo.gl/8p3tPZ>. Acesso em: 04 dez. 2018.

MAGALHÃES, Alline et al. Nas farmácias, venda de remédio subiu $42 \%$ em cinco anos. Disponível em: <http://infograficos.estadao.com.br/focas/tanto-remedio-paraque/checkup-1.php>. Acesso em: 06 dez. 2018.

MICHAEL, J. W.; COHN, Alan; BUTCHER, Jared R. BlockChain technology. The Journal, 2018.

MICROSOFT. Understanding Public Key Cryptography. 2014. Disponível em: <https://docs.microsoft.com/en-us/previous-versions/tn- archive/aa998077(v=exchg.65)>. Acesso em: 24 nov. 2018.

NAKAMOTO, Satoshi. Bitcoin: A Peer-to-Peer Electronic Cash System. [s.1.]: Bitcoin, 2008. 9 p. Disponível em: <https://bitcoin.org/bitcoin.pdf>. Acesso em: 24 nov. 2018.

ORGANIZAÇÃO MUNDIAL DE SAÚDE. Prequalification of medicines by WHO. 2013. Disponível em: <https://www.who.int/news-room/fact- sheets/detail/prequalification-ofmedicines-by-who>. Acesso em: 08 dez. 2018.

PINTO, Vanusa Barbosa. Armazenamento e distribuição: o medicamento também merece cuidados. Uso Racional de Medicamentos: fundamentação em condutas terapêuticas e nos macroprocessos da Assistência Farmacêutica, Brasília, v. 1, n. 12, jul. 2016. Disponível em: <https://goo.gl/Qdsa2n>. Acesso em: 9 dez. 2018.

PRADO, Jean. O que é blockchain: indo além do bitcoin. 2017. Disponível em: <https://goo.gl/Z6EZAS/>. Acesso em: 23 nov. 2018.

RANDE, Tássia Martins. Smart Contracts: o que são e como funcionam? 2018. Disponível em: <https://goo.gl/fexWzF>. Acesso em: 16 nov. 2018.

VENEGAS, Percy. Economy Monitor Guide to Smart Contracts: Blockchain Examples. [s.l.]: Economy Monitor, 2017. 43 p. Disponível em: 〈https://goo.gl/cqYngV〉. Acesso em: 17 nov. 2018.

YLI-HUUMO, Jesse et al. Where is current research on blockchain technology? - a systematic review. PloS one, v. 11, n. 10, p. e0163477, 2016 